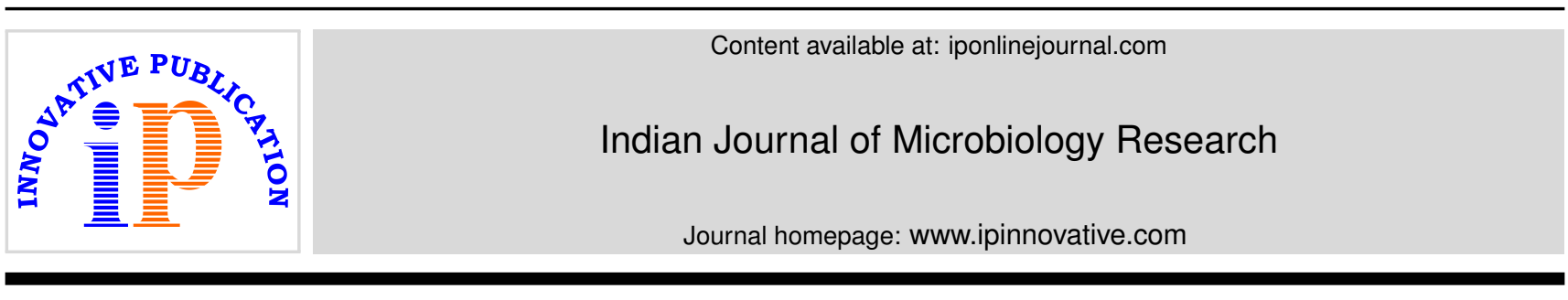

Original Research Article

\title{
Molecular characterization of metallo-beta-lactamase producers among carbapenem resistant Pseudomonas aeruginosa isolated from cases of hospital acquired infections in a tertiary care hospital, Bengaluru
}

\author{
Ashwini Sondakar', Sneha K Chunchanur², Ambica Rangaiah², \\ Sathyanarayan Muthur Shankar ${ }^{2, *}$ \\ ${ }^{1}$ Dept. of Microbiology, Belgaum Institute Medical Sciences, Belagavi, Karnataka, India \\ ${ }^{2}$ Dept. of Microbiology, Bangalore Medical College \& Research Institute, Bengaluru, Karnataka, India
}

\section{A R T I C L E I N F O}

Article history:

Received 04-02-2020

Accepted 13-03-2020

Available online 20-07-2020

Keywords:

Metallobetalactamases

Carbapenemase

Pseudomonas aeruginosa

\begin{abstract}
A B S T R A C T
Background: Pseudomonas aeruginosa is an important cause of multidrug-resistant nosocomial infections The knowledge of resistance mechanisms in Pseudomonas is an important issue for antimicrobial treatment. Therefore, the objective was to detect carbapenems resistance in P. aeruginosa by phenotypic methods and genes (blaIMP and blaVIM) coding for carbapenemase resistance.

Materials and Methods: The study conducted in the Department of Microbiology, BMCRI, Bengaluru. 91 samples from the patients hospitalised for 48 hours and more were processed. Carbapenem resistant P. aeruginosa (CRPA) were identified by biochemical tests and by Kirby Bauer Disk diffusion method as per CLSI guidelines. Those carbapenem resistant isolates were further subjected to two MBL detecting phenotypic tests- Modified Hodge Test (MHT) and Combined disk Test (CDT) by using imipenem and imipenem/ EDTA disk and MBL genes (blaIMP and blaVIM) were identified by PCR.

Results: 91 clinical isolates were identified as CRPA, 92.3\% isolates were positive by CDT whereas only $13.2 \%$ of isolates showed positive by MHT method indicating MBL production. Among 91 strains, $19.04 \%$ strains were harbouring blaVIM and $1.2 \%$ strain harbouring blaIMP gene.

Conclusion: The detection of MBL-producing P. aeruginosa help in appropriate antimicrobial therapy and avoid development and dissemination of these strains. Hence routine detection of MBL production in P. aeruginosa should be undertaken. All CRPA isolates should be routinely screened for MBL production using CDT and positive isolates should be confirmed by PCR. MHT test had low sensitivity. To understand the epidemiology, there is a need of genetic analysis and typing of MBL enzymes.
\end{abstract}

(C) 2020 Published by Innovative Publication. This is an open access article under the CC BY-NC license (https://creativecommons.org/licenses/by-nc/4.0/)

\section{Introduction}

Pseudomonas aeruginosa is a Gram-negative bacterium with the ability to persist in both community and hospital settings. It is an important nosocomial pathogen with emerging resistance to many effective groups of antibiotics through both intrinsic and acquired resistance mechanisms. Carbapenems are the drug of choice for infections caused by Pseudomonas aeruginosa including other Gram-negative organisms. ${ }^{1}$ Global emergence of

\footnotetext{
* Corresponding author.

E-mail address: drsathyams@gmail.com (S. M. Shankar).
}

carbapenem resistance Pseudomonas aeruginosa (CRPA) including resistance to beta-lactams, Aminoglycosides, and Fluoroquinolones represents an extraordinary threat to public health. The major mechanisms of carbapenem resistance in Pseudomonas aeruginosa include carbapenem hydrolyzing enzymes i.e. carbapenemase, decrease outer membrane permeability and alteration penicillin-binding proteins. ${ }^{2}$ However, the clinical use of these antimicrobials is under threat with the emergence of carbapenemases, particularly Metallo- 3 -lactamases (MBLs). ${ }^{2}$ MBL belongs to Ambler class $\mathrm{B}$, and these enzymes can hydrolyze a wide variety of beta-lactam agents, such as penicillin, 
cephalosporins, and carbapenems. They require zinc for their catalytic activity and are inhibited by metal chelators, such as EDTA and thiol-based compounds. ${ }^{3}$ MBLs in P. aeruginosa were identified in 1991 in Japan $^{4}$ and since then have been described from various parts of the world, including Asia, Europe, Australia, South America, and North America ${ }^{4,5}$ whereas in India bla $V I M$, bla ${ }_{I M P}$ and bla ${ }_{N D M}$ genes are frequently encountered in P. aeruginosa. ${ }^{6}$

The genes responsible for the production of MBLs are typically part of an integron structure and are carried on transferrable plasmids but can also be part of the chromosome. ${ }^{7}$ Due to integron-associated gene cassettes, MBL producing $\mathrm{P}$. aeruginosa isolates are often resistant to different groups of antimicrobial agents, which can be transferred to another Gram-negative bacteria. ${ }^{8}$

MBLs in Carbapenem-resistant Pseudomonas aeruginosa can be detected by different phenotypic methods and these methods are based on the ability of metal chelators to inhibit the activity of MBLs such as EDTA and thiol-based compounds. ${ }^{9}$ These include Combined Disk Test (CDT) ${ }^{9}$ using EDTA with imipenem (IPM), Modified Hodge test (MHT), MBL Epsilometer test (E-test) and EDTA disk potentiation test. ${ }^{10,11}$ A PCR detection assay is considered as a gold standard method for the detection of MBL producers. ${ }^{12}$ Because of the increasing rate of resistance to the carbapenems, the treatment of infections produced by MBLs producing $\mathrm{P}$. aeruginosa is becoming critical.

Hence the present study was undertaken to detect presence of MBLs among Carbapenem-Resistant Pseudomonas aeruginosa (CRPA) isolates by phenotypic methods like MHT and Combined Disk Test (CDT) by using Imipenem (IPM)- Ethylene diamine tetraacetic acid (EDTA) and to identify the MBL genes (blaVIM and blaIMP) coding for carbapenemase resistance by conventional PCR in a tertiary care hospital in Bengaluru.

\section{Materials and Methods}

This study was conducted over a period of one year from January 2018 to December 2018 at a tertiary hospital, BMCRI, Bengaluru. Ethical clearance was obtained from the institutional ethical committee. This study included 91 non-consecutive clinical samples from patients hospitalized for 48 hours and more, received for culture and sensitivity in the Department of Microbiology.

All the clinical specimens were subjected to direct microscopy, growth on culture media and series of tests for identification of $\mathrm{P}$. aeruginosa. These isolates were subjected to antimicrobial susceptibility testing by KirbyBauer disk diffusion method according to CLSI guidelines. ${ }^{13}$ The antibiotics tested include amikacin $(30 \mu \mathrm{g})$, ciprofloxacin $(5 \mu \mathrm{g})$, ceftazidime $(30 \mu \mathrm{g})$, piperacillintazobactam $(100 / 10 \mu \mathrm{g})$, imipenem $(10 \mu \mathrm{g})$, meropenem (MEM-10 $\mu \mathrm{g}$ ), aztreonam (30 $\mu \mathrm{g})$, colistin (10 $\mu \mathrm{g})$ and polymyxin-B (300 units). All the disks were obtained commercially (Hi-Media Laboratories Limited. Mumbai, India). ATCC strain of Pseudomonas aeruginosa 27853 is used as control.

All isolates resistant to imipenem or meropenem or ceftazidime or any two of them were considered as probable MBL producer. All positive isolates were further tested by two phenotypic tests for the MBL detection, described as follows.

\subsection{Imipenem-Ethylene diamine tetraacetic acid combined disc test}

A lawn culture of test isolate was prepared. Allowed to dry for five minutes. Two imipenem $(10 \mu \mathrm{g})$ discs, one with 0.5 M EDTA and other a plain imipenem disc, were placed on the surface of agar plates approximately $30 \mathrm{~mm}$ apart. The plates were incubated overnight at $37^{\circ} \mathrm{C}$ for $16-18 \mathrm{~h}$. An increase in zone diameter of $>7 \mathrm{~mm}$ around the imipenemEDTA disc in comparison to imipenem disk alone indicates the production of MBL. ${ }^{9}$

\subsection{Modified Hodge Test (MHT)}

A saline suspension of a 0.5 McFarland standard of E. coli ATCC 25922 was prepared and diluted 1:10 and lawn inoculated on Muller Hinton Agar (MHA). The plate was allowed to dry for 3-10 minutes. An imipenem (10 $\mu \mathrm{g})$ disk was placed at the center and 3-5 colonies of test organisms were inoculated in a straight line drawn out from the edge of the disk. A known NDM positive strain was used as Positive control and incubated overnight at $35^{\circ} \mathrm{C}$ for $20-24 \mathrm{~h}$. the presence of a distorted zone of inhibition or cloverleaf type of indentation at the intersection of the test organism and E. coli. within the zone of inhibition of the IPM disk was interpreted as a positive result. ${ }^{10,11}$

\subsection{PCR for carbapenem encoding gene}

Isolates tested positive in the phenotypic test are subjected to conventional PCR for detection genes coding for MBLs. The DNA extraction was done by the crude method (boiling method). Freshly subcultured colonies were suspended in 50 $\mu \mathrm{l}$ of PCR grade water, heated to $99^{\circ} \mathrm{C}$ for 10 minutes in a water bath and kept at room temperature for 5 minutes. The suspension is centrifuged at $14000 \mathrm{rpm}$ for $1 \mathrm{~min}$ at $4^{0} \mathrm{C}, 5$ $\mu 1$ of supernatant was used as the template for a $50 \mu 1 \mathrm{PCR}$ reaction. PCR was performed by using previously designed primers (Sigma-Aldrich, Bengaluru) for bla ${ }_{V I M}$, and bla $I M P$ (Table 1) ${ }^{14}$

Amplification was performed in $50 \mu 1$ PCR mixture consisting of master mix $(25 \mu \mathrm{l})$ - Taq DNA polymerase 2x master mix RED $1.5 \mathrm{mM} \mathrm{MgCl2}$ (Synergy Scientific Service PVT. LTD), PCR grade water $(18 \mu \mathrm{l})$, Primer bla $_{V I M}$, and bla IMP $_{\text {F' and R' (1 }} \mu \mathrm{l}$ each) and DNA $(5 \mu \mathrm{l})$.

DNA was amplified in a Master cycler Eppendorf under the following conditions (Table 1) and PCR products 
were kept at $4^{0} \mathrm{C}$. Known bla ${ }_{V I M}$, and bla ${ }_{I M P}$, producing laboratory strain Pseudomonas aeruginosa was used as the positive control. Pseudomonas aeruginosa ATCC 27853 reference strain was used as the MBLgene's negative strain. Cycling conditions for MBL (blaVIM and blaIMP) gene were described (Table 2). ${ }^{12}$

The PCR products were analyzed by gel electrophoresis with $2 \%$ agarose gel in TAE (tris-acetate buffer) buffer with $1.5 \mu 1$ ethidium bromide and were visualized and photographed under ultraviolet illumination.

\section{Results}

Among ninety-one CRPA isolates included in the study $70.3 \%$ were from the pus, $12.08 \%$ from blood and body fluids, $6.5 \%$ from urine, $3.3 \%$ from respiratory samples and $3.29 \%$ from others (Corneal and ENT). Distribution of isolates according to wards as follows, General surgery (42.8\%), burns and plastic surgery $(27.4 \%)$, ICUs $(9.8 \%)$, orthopedics $(4.3 \%)$, Nephro-urology (4.3\%) and others ENT and ophthalmic (2.19\%).

The antimicrobial susceptibility testing of CRPA isolates showed resistance to other antimicrobials including ceftazidime and cefepime(95.6\% each), ciprofloxacin and levofloxacin (91.2\% each), piperacillin(94.5\%), ticarcillin (96.7\%), piperacillin-tazobactam(86.8\%), $\operatorname{amikacin}(83.5 \%)$, gentamicin $(86.8 \%)$, aztreonam(80.2\%), whereas only $12.08 \%$ isolates were resistant to colistin (Figure 1).

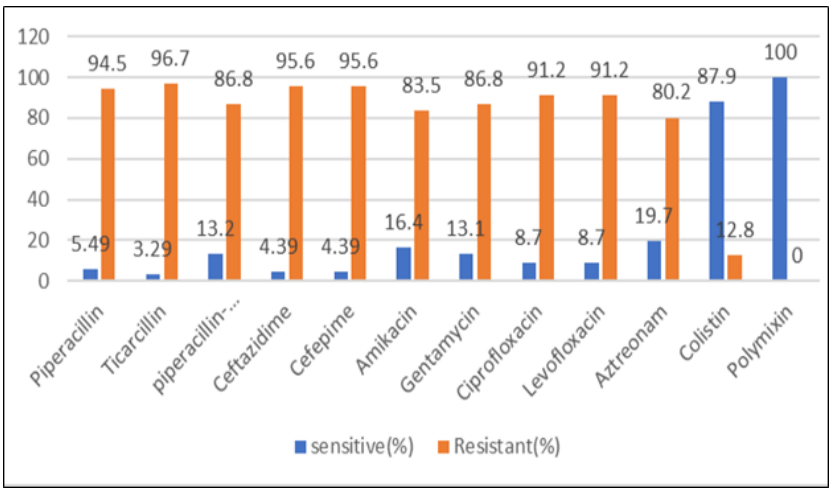

Fig. 1: Antimicrobial susceptibility test in Carbapenem resistant pseudomonas aeruginosa (CRPA) isolates. Antimicrobial susceptibility test in carbapenem resistant pseudomonas aeruginosa (CRPA) isolates

\subsection{MBL phenotypic screening tests}

Among the two phenotypic tests, Combined Disc Test using imipenem and EDTA was positive in 84 isolates and Modified Hodge Test was positive in only 12 isolates. In this study, CDT showed high sensitivity (92.3\%) while MHT was least sensitive (13.18\%) (Figure 2).

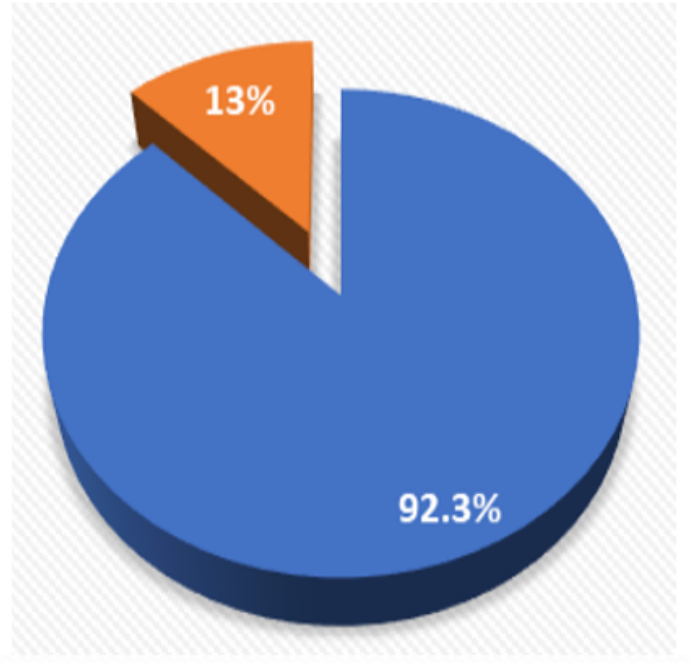

Combined Disk Test Modified Hodge Test

Fig. 2: MBL phenotypic screening tests

\subsection{PCR for carbapenem encoding gene}

Positively tested isolates by MBL phenotypic screening tests are further confirmed by conventional PCR. Out of 84 isolates, 16 isolates carried bla $V I M(19.04 \%)$ and one isolate carried bla $I M P$ gene (1.19\%). (Figures 3, 4 and 5)

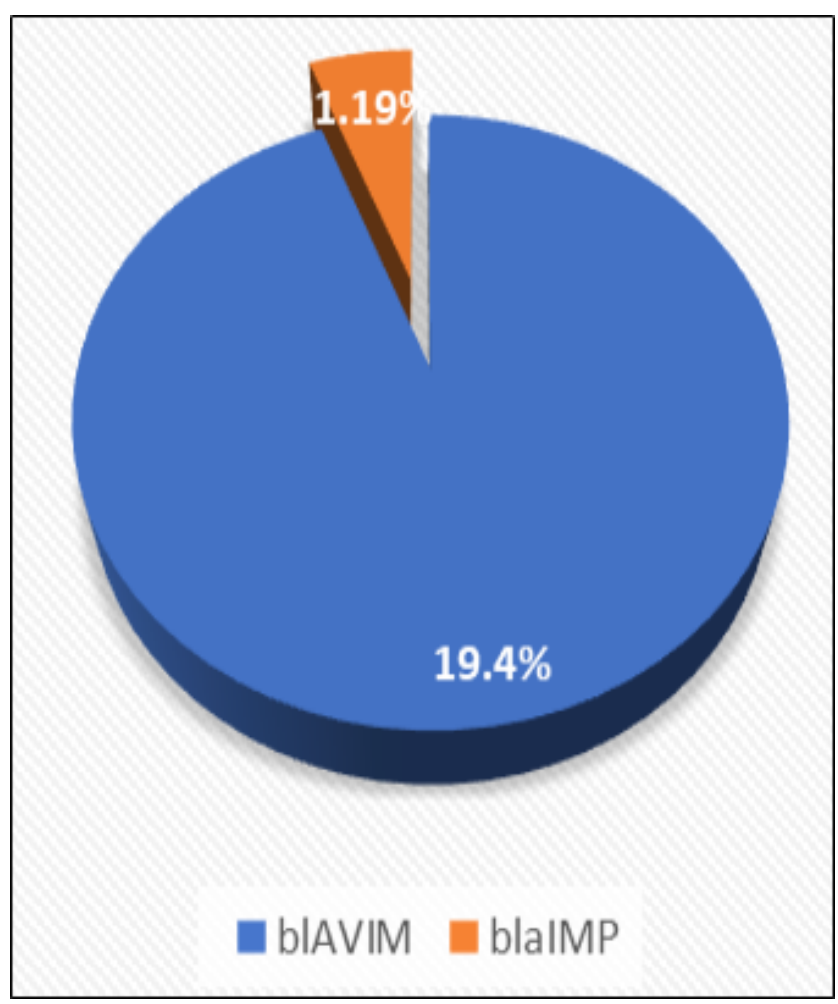

Fig. 3: MBL genes coding for Carbapenen resistance 
Table 1: Primer sequence used in PCR and their product size as follows. ${ }^{14}$

\begin{tabular}{|c|c|c|c|}
\hline Primer & \multicolumn{2}{|c|}{ Primer sequence (5'-3') } & Product size (base pairs) \\
\hline bla VIM-F & \multicolumn{2}{|c|}{ TTT GGT CGC ATA TCG CAACG } & 500 \\
\hline bla VIM-R & \multicolumn{2}{|c|}{ CCA TTC AGC CAG ATC GGC AT } & 500 \\
\hline bla IMP-F & \multicolumn{2}{|c|}{ GTT TAT GTT CAT ACW TCG } & 432 \\
\hline bla IMP-R & \multicolumn{2}{|c|}{ GGT TTA AYA AAA CAA CCA $\mathrm{C}(\mathrm{W}=\mathrm{A}$ or $\mathrm{T} ; \mathrm{Y}=\mathrm{C}$ or $\mathrm{T})$} & 432 \\
\hline Steps in PC & & VIM & IMP \\
\hline Initial DNA & & $940 \mathrm{C} 5 \mathrm{~min}$ & $940 \mathrm{C} 5 \mathrm{~min}$ \\
\hline Final DNA $\mathrm{c}$ & & $950 \mathrm{C} 30 \mathrm{sec}$ & $950 \mathrm{C} 30 \mathrm{sec}$ \\
\hline Primer anne & & $660 \mathrm{C} 1 \mathrm{~min}$ & $450 \mathrm{C} 1 \mathrm{~min}$ \\
\hline Primer exten & & $720 \mathrm{C} 30 \mathrm{sec}$ & $720 \mathrm{C} 26 \mathrm{sec}$ \\
\hline Final extensi & iperature & $720 \mathrm{C} 10 \mathrm{~min}$ & $720 \mathrm{C} 10 \mathrm{~min}$ \\
\hline Cycles & & 30 & 30 \\
\hline
\end{tabular}

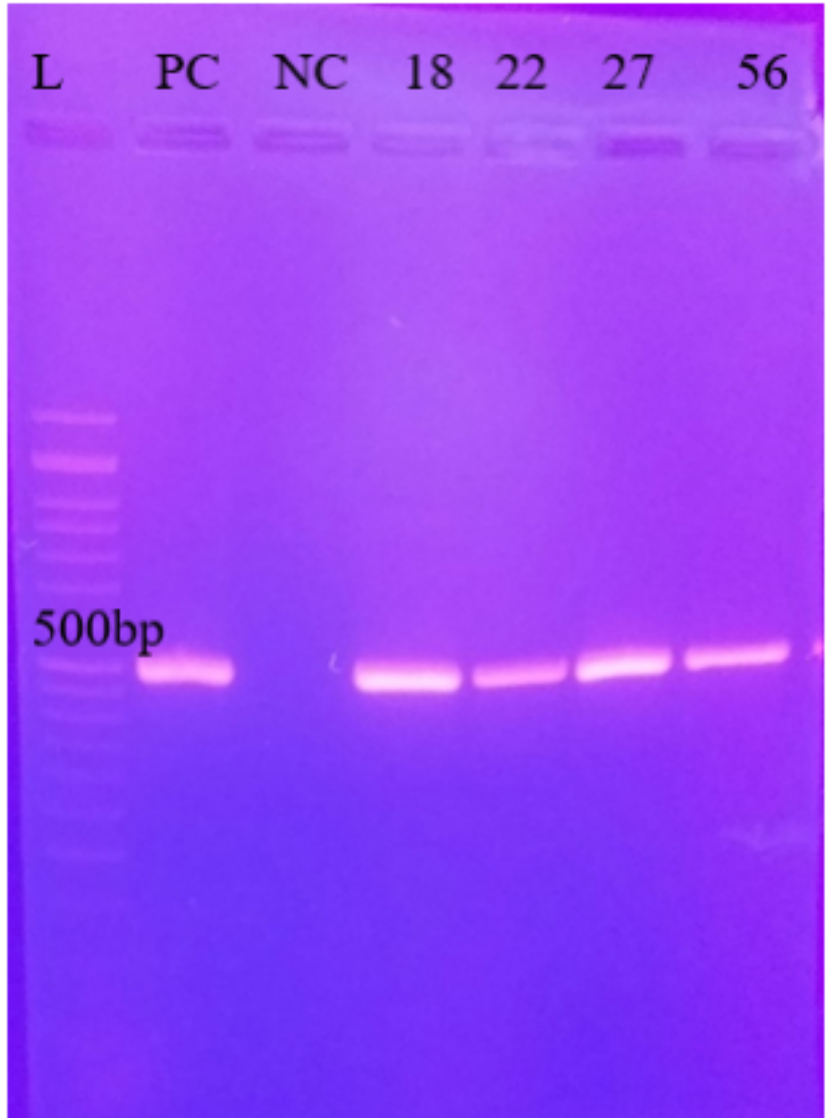

Fig. 4: Agarose gel run for blaVIM

\section{Discussion}

Antimicrobial resistance is a significant route cause of healthcare-associated infections all over the world. Carbapenem has been used as the last choice of treatment of many Gram-negative organisms. In the current study, we have included carbapenem-resistant P. aeruginosa isolates. Carbapenem resistance among Gram-negative bacteria has

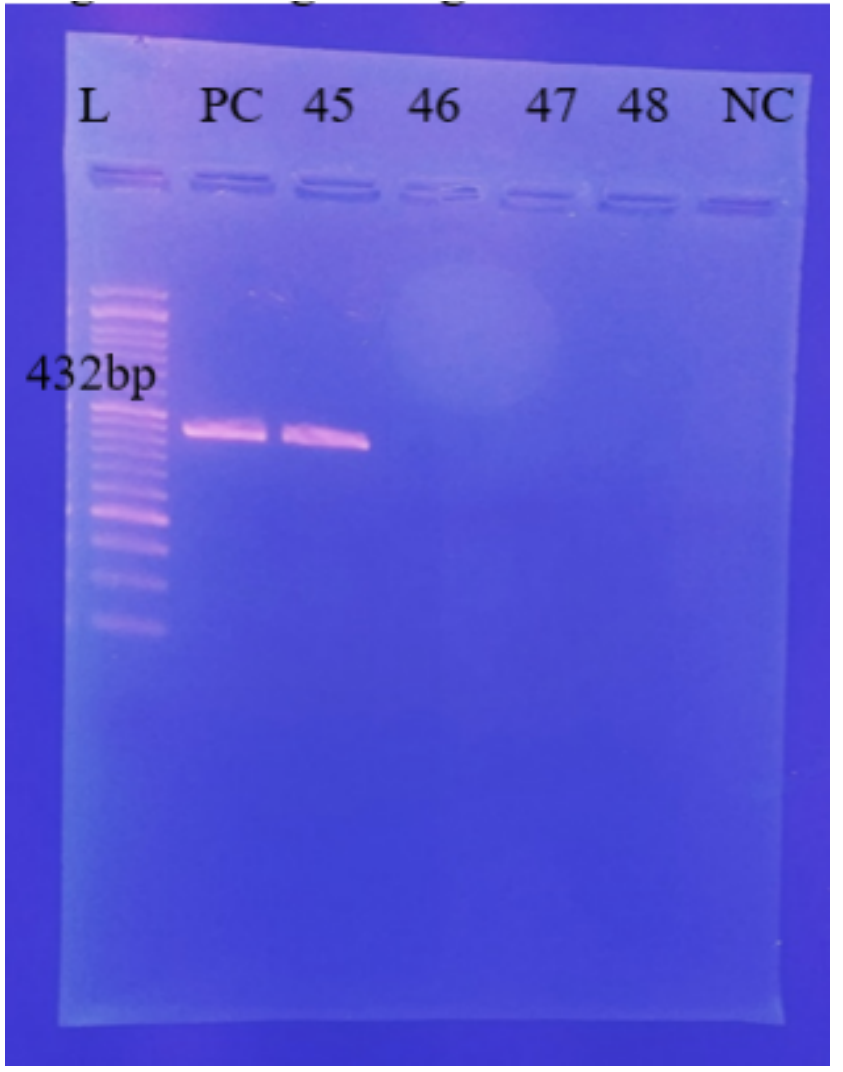

Fig. 5: Agarose gel run for blaIMP

been increased in recent years in the Indian subcontinent and MBL producing isolates have emerged worldwide and are associated with outbreaks in health care settings over the past few years.

In this study, most of the carbapenem-resistant Pseudomonas aeruginosa isolates were from pus $(70.3 \%)$ and from general surgery ward (42.8\%) and most of them were resistant to other class of antibiotics 
such as Anti-pseudomonal Penicillin's, Aminoglycosides, Fluoroquinolones, Cephalosporins, and Aztreonam. This indicates the concomitant presence of other beta-lactamases. Polymyxins showed susceptibility of $87.9 \%(n=91)$ which was high compared to other classes of antibiotics and hence considered as a treatment option of CRPA isolates.

In this study, MBL production was screened by the Modified Hodge test and Combined Disk Test by using Imipenem-EDTA. Out of 91 isolates, MHT was positive in 84 isolates $(92.3 \%)$ and MHT was positive in 12 isolates (13.18\%). PCR detected MBL genes $b^{2} a_{V I M} / b_{l a} a_{I M P}$ in $20.2 \%$ (bla ${ }_{V I M}-16$ and bla $_{I M P}-1$ ) among those positive isolates by phenotypic tests. This coincides with a high prevalence of bla ${ }_{V I M}$ among Pseudomonas isolates, a study by Amudhan $\mathrm{M}$ et al. ${ }^{14}$ The remaining 71 isolates that were MBL phenotypic test positive, were negative for both MBL genes $\left(\mathrm{bla}_{V I M} / \mathrm{bla}_{I M P}\right)$ suggesting the presence of other MBL genes such as SIM-1, GIM-1, NDM-1 or SPM-1.

Despite the good performance of inhibitor-based methods (Combined disk test using IMP-EDTA) for the detection of MBL, false-positive results were found, hence this is not considered as a specific test. False positives may be due to EDTA which acts on the membrane of a bacterial cell and increases cell permeability. MHT also showed very low sensitivity. Hence the results of the MBL phenotypic tests must be interpreted cautiously.

The overall $\mathrm{bla}_{V I M} / \mathrm{bla}_{I M P}$ production among study isolate was $20.2 \%$ among them, bla $a_{V I M}$ was found in 16 isolates whereas bla IMP $_{\text {Was }}$ found in one isolate. In Asia MBL genes, bla ${ }_{V I M}$ and bla $_{I M P}$ are prevalent and bla $_{I M P}$ is found in Japan, Korea, China, Taiwan and Iran. ${ }^{15-17}$

The prevalence of MBL genes in India ranges from 7$65 \%$ among $P$. aeruginosa. In a study from India showed, the rate of MBL production was $24.5 \%$ among $61 \mathrm{P}$. aeruginosa isolates and bla $\mathrm{a}_{V I M}$ was the most common. Another study from India also reported blaVIM -2 from P. aeruginosa. ${ }^{18}$ In a nationwide survey conducted to characterize $301 \mathrm{MBL}$ producing Pseudomonas species in 10 medical centers from India, the MBL genes were detected in $18.9 \%$ isolates. ${ }^{19}$ There are fewer data available on the prevalence and distribution of MBL among Indian isolates.

In this study, MBL phenotypic tests were positive in 92.3\% isolates and among them, 20.2\% isolates were carrying bla $_{V I M} / \mathrm{bla}_{I M P}$ genes indicating carbapenemase production. So, early detection of MBL production in Carbapenem-resistant Pseudomonas aeruginosa will not only help in treating the infections caused by them adequately and also help in preventing the spread of multidrug resistance to other Gram-negative strains. Therefore, all clinical isolates that are resistant to carbapenem must be screened for MBL production by using simple phenotypic tests and confirmed by the MBL Epsiolometer test (E-test) or by PCR if possible.

To conclude, carbapenem resistance in P. aeruginosa is chiefly mediated by Metallo-beta-lactamase production.
Among the two phenotypic tests performed in this study, CDT was more sensitive compared to the Modified Hodge Test in the screening of MBL production. Genotypically, the common MBL gene -found was blaVIM compared to blaIMP. Therefore, screening for MBL production in microbiology laboratories is crucial for optimal treatment of patients, particularly hospitalized patients and also to prevent the possible spread of resistance to other Gram-negative organisms because of their broad-spectrum drug resistance which creates a therapeutic challenge to clinicians. Finally, to understand the epidemiology, there is a need for genetic analysis and also typing of Metallo- 3 lactamase enzymes.

\section{Source of Funding}

None.

\section{Conflict of Interest}

None.

\section{References}

1. Singh SP, Shariff M, Barua T, Thukral SS. Comparative evaluation of phenotypic tests for identifi cation of Metallo beta-lactamases producing clinical isolates of Pseudomonas aeruginosa. Indian J Med Res. 2009;129:713-5.

2. Livermore DM, Woodford N. Carbapenemases: a problem in waiting? Curr Opin Microbiol. 2000;3(5):489-95.

3. Manoharan A, Chatterjee S, Mathai D. Detection and characterization of metallo beta lactamases producing Pseudomonas aeruginosa. Indian J Med Microbiol. 2010;28(3):241.

4. Watanabe M, Iyobe S, Inoue M, Mitsuhashi S. Transferable imipenem resistance in Pseudomonas aeruginosa. Antimicrob Agents Chemother. 1991;35(1):147-51.

5. Pitout JDD, Gregson DB, Poirel L, McClure JA, Le P, Church DL. Detection of Pseudomonas aeruginosa Producing Metallo- Lactamases in a Large Centralized Laboratory. J Clin Microbiol. 2005;43(7):3129-35.

6. Brahmadathan $\mathrm{KN}$, Gladstone $\mathrm{P}$, Rajendran $\mathrm{P}$. Incidence of carbapenem resistant nonfermenting gram negative bacilli from patients with respiratory infections in the intensive care units. Indian J Med Microbiol. 2005;23(3):189.

7. Poirel L, Nordmann P. Acquired Carbapenem-Hydrolyzing BetaLactamases and their Genetic Support. Curr Pharm Biotechnol. 2002;3(2):117-27.

8. Nordmann P, Poirel L. Emerging carbapenemases in Gram-negative aerobes. Clin Microbiol Infect. 2002;8(6):321-31.

9. Saderi H, Karimi Z, Owlia P, Bahar MA, Rad SM. Phenotypic detection of metallo-beta-lactamase producing Pseudomonas aeruginosa strains isolated from burned patients. Iran J Pathol. 2008;3:20-4.

10. Yong D, Lee K, Yum JH, Shin HB, Rossolini GM, Chong Y. Imipenem-EDTA Disk Method for Differentiation of Metallo- Lactamase-Producing Clinical Isolates of Pseudomonas spp. and Acinetobacter spp. J Clin Microbiol. 2002;40(10):3798-3801.

11. Lee K, Lim YS, Yong D, Yum JH, Chong Y. Evaluation of the Hodge Test and the Imipenem-EDTA Double-Disk Synergy Test for Differentiating Metallo- -Lactamase-Producing Isolates of Pseudomonas spp. and Acinetobacter spp. J Clin Microbiol. 2003;41(10):4623-9.

12. Sheikh AF, Rostami S, Jolodar A, Tabatabaiefar MA, Khorvash F, Saki A, et al. Detection of Metallo-Beta Lactamases Among Carbapenem-Resistant Pseudomonas aeruginosa. Jundishapur J 
Microbiol. 2014;7(8). do1:10.5812/1]m.12289.

13. Performance Standards for Antimicrobial Susceptibility Testing: Twentieth Informational Supplement M100-S20-U. Wayne, PA: CLSI; 2018.

14. Amudhan MS, Sekar U, Kamalanathan A, Balaraman S. blaIMP and blaVIM mediated carbapenem resistance in Pseudomonas and Acinetobacter species in India. J Infect Dev Ctries. 2012;6(11):75762.

15. Dong $\mathrm{F}, \mathrm{Xu} \mathrm{X}$, Song WLP, Yu S, Yang Y. Characterization of multidrug-resistant and Metallo-beta-lactamase-producing Pseudomonas aeruginosa isolates from a pediatric clinic in China. Chin Med J. 2008;121(17):1611-6.

16. Peymani A, Nahaei MR, Farajnia S, Hasani A, Mirsalehian, Sohrabi $\mathrm{N}$, et al. High prevalence of Metallo- $\beta$ lactamase- producing Acinetobacter baumannii in a teaching hospital in Tabriz, Iran. Jpn J Infect Dis. 2011;64:69-71.

17. Franco MRG, Caiaffa-Filho HH, Burattini MN, Rossi F. Metallo-betalactamases among imipenem-resistant Pseudomonas aeruginosa in a Brazilian university hospital. Clinics. 2010;65(9):825-9.

18. Toleman MA, Vinodh H, Sekar U, Kamat V, Walsh TR. blaVIM2-Harboring Integrons Isolated in India, Russia, and the United States Arise from an Ancestral Class 1 Integron Predating the Formation of the $3^{\prime}$ Conserved Sequence. Antimicr Agents Chemother. 2007;51(7):2636-8.

19. Castanheira M, Bell JM, Turnidge JD, Mathai D, Jones RN Carbapenem Resistance among Pseudomonas aeruginosa Strains from India: Evidence for Nationwide Endemicity of Multiple Metallo- $\beta$-Lactamase Clones (VIM-2, $-5,-6$, and -11 and the Newly Characterized VIM-18). Antimicr Agents Chemother. 2009;53(3):1225-7.

\section{Author biography}

Ashwini Sondakar Senior Resident

Sneha K Chunchanur Assistant Professor

Ambica Rangaiah Professor and Head

Sathyanarayan Muthur Shankar Assistant Professor

Cite this article: Sondakar A, Chunchanur SK, Rangaiah A, Shankar SM. Molecular characterization of metallo-beta-lactamase producers among carbapenem resistant Pseudomonas aeruginosa isolated from cases of hospital acquired infections in a tertiary care hospital, Bengaluru. Indian J Microbiol Res 2020;7(2):212-217. 